\title{
S32. Assessment of quality of life in psychiatry (supported by an educational grant from Astra S)
}

The Meaning of Psychological Well-Being CD Ryff

Department of Psychology, University of Wisconsin Madison, Wisconsin, USA 53706

Deeply entrenched conventions have defined mental health as the absence of illness. Healthy functioning is thus equated with not being psychologically troubled in some way. This prevailing formulation never gets to the heart of wellness, where the task is to define mental health as the presence of the positive. A multidimensional conception of psychological well-being will be presented, which is grounded in the integration of theories from developmental psychology, clinical psychology, and the mental health literature. Six key aspects of well-being will be described: self-acceptance, environmental mastery, purpose in life, autonomy, positive relations with others, and personal growth. A program of empirical research investigating this formulation will be summarized. Included are studies of age, gender, and cultural differences in the various aspects of well-being. Augmenting these descriptive inquiries are studies that explain human variation in positive functioning through a focus on life events and experiences. How individuals interpret and give meaning to their experiences, via social comparison processes and reflected appraisals, is an important part of this research agenda.
THE WORLD HEALTH ORGANIZATION QUALITY OF LIFE ASSESSMENT INSTRUMENT: METHODOLOGY AND EARLY DATA

M Amir

Department of Behavioral Sciences, Ben-gurion University of the Negev, POB 653, 84105 Beer-Sheva, Israel

The WHO Division of Mental Health is developing an instrument for the measurement of quality of life (the WHOQOL), which allows the inquiry into the perception of individuals of their own position in life in the context of the culture and value systems in which they live and in relation to their goals, expectations, standards and concerns.

This has been carried out in a project involving 15 centres in different cultural settings, across which it will maintain comparability. A pilot version of the instrument has been piloted on 300 subjects in each site and the data is now being analyzed. Versions of the instrument will begin to be field tested at the end of this year for defining their psychometric characteristics. Some data from the pilot testing will be presented in the full paper 


\section{THE ASSESSMENT OF QUALITY OF LIFE IN SCHIZOPHRENIA \\ Anders Rylander \\ Astra Arcus, CNS Clinical Research, S-151 85 Södertälje, Sweden}

Since schizophrenia is a chronic disease that affects many aspects of life the need for assessing the impact of the disease and its treatment upon quality of life seems to be of clinical importance. Quality of life studies in schizophrenic patients will probably also play a role in the pharmacoeconomic evaluation of different interventions. In spite of this there are relatively few studies on this subject, especially studies dealing with the impact of antipsychotics on quality of life.

Available studies that will be reviewed indicate that schizophrenic patients, as expected, have a lower quality of life than samples from a general population. By identifying domains of particular importance to the individual, treatment programs could be optimized and good working alliances created.

Very few studies on the impact of antipsychotic drugs have been published. The methodology and results of these studies will be presented.

In order to assess the quality of life of schizophrenic patients there is probably necessary to use a set of rating scales. The need for relevant disease-specific rating scales will be also be highlighted.

\author{
THE QUALITY OF LIFE AND DISABLEMENT ASSESSMENTS \\ THEORETICAL AND PRACTICAL RELATIONS \\ Jorley \\ Division of Mental Health, World Health Organization, 1211 Geneva \\ 27, Switzerland
}

The WHOQOL group defines Quality of Life as the "individuals' perception of their position in life in the context of the culture and value systems in which they live and in relation to their goals, expectations, standards and concerns". This definition can be viewed as person's "subjective" perception, whereas disablements according to ICDH is "objective".

ICDH (International Classification of Impairments, Disabilities, and Handicaps) examines the "objective" consequences of disorders in three planes as impairments: structural or functional deficits; disabilities: dysfunction in activities; and handicaps: societal hindrances on people because of their impairments or disabilities. This classification has been used for thirteen years and found applicable and useful in the area of mental health. While some impairments (such as intellectual or cognitive impairments) are specific to mental health care, disabilities and handicaps are not different for "mental" and "physical" disorders .

The assessments of "quality of life" and "disablements" therefore, reflect complementary views of the same universe from two different viewpoints (that of the patient and that of an expert). The Division of Mental Health of WHO has developed assessment instruments for measuring quality of life and disablements in different cultures. This paper deals with the links between these two approaches and defines the methodology employed to identify assessment domains that will be sensitive to the cultural setting in which it is applied, while maintaining comparability of scores across different cultural settings.
QUALITY OF LIFE: ASSESSING THE INDIVIDUAL.

CA O'Boyle.

Department of Psychology, Medical School, Royal college of Surgeons in Ireland, St. Stephen's Green, Dublin 2.

The Quality of Life (QoL) construct may be viewed as a paradigm shift in medicine since it atters the focus of attention from symptoms to functioning. This holistic focus more clearty establishes the patient at the centre of attention and subsumes many of the traditional measures of outcome. Modern psychiatry with its orientation towards rehabilitation and care within community seltings has adopted a simitar perspective to that which, in other within communky sellings has adopted a similar perspective to that which, in othe disciplines, is called QoL. A broader biopsychosocial perspective incorporating, the QoL construct may result in treatments which are more closely attuned to the needs of patients and their families.

Despite the increasing importance of $\mathrm{QOL}$, there is little agreement regarding what should be assessed or the manner in which information should be collected. It is generally agreed that diseases and their treatment impact on such areas of functioning as mobility, mood, life satisfaction, cognition, and ability to fulfil occupational, social and family roles and most QoL instruments include measures of each of these domains. However, there are a variety of approaches to assessment and there exist a bewildering array of scales, questionnaires and interviews all purporting to measure Ool such methods are generally developed using grouped data and grouped data and the hems and sconing systems may not be appropriate to individua the factors which are mportant to him or her, rate their current level of function or satisfaction and provide an indication of the relative importance of each nominated component.

This paper discusses the individual nature of QoL and illustrates the significant difficulties in definition, measurement and interpretation which must be addressed before such measures can be used as rellable and valid Indicators. It is argued that the individual perspective of the patient must be incorporated into any assessment of QoL and the scope of assessment mlght usefully include the QoL of the family or carer(s). This paper develops the argument that $Q 0 L$ is best defined as an individual phenomenon refiecting the perceptions of individual it is proposed that the appropriate defintion of $\mathrm{O}$ is is that which perceptons of the indvidual determines it to be. Measures which have incorporated the Individua perspective will be reviewed and the Schedule for the Evaluation of Individual Quality of Life (SEIQOL) will be described in detall. The recent development of a Direct Weighting Procedure for measuring the relative importance, to patients, of particular life areas will also be described. The application of these measures in a variety of patient populations will be described and the advantages and disadvantages of the individual approach will be discussed. The implications of the individual approach for proxy Judgments of QoL will be highlighted. 